\section{Reduction of plasma leptin during a short-term fast, an oral glucose tolerance or a meal test can be a misleading bias in clinical studies}

Dear Sir,

A substantial number of papers have been published in the last few years on leptin and on its regulation by insulin $[1,2]$. This has stimulated us to examine changes of plasma leptin concentrations in response to different paradigms: a 75-g oral glucose challenge, a standardised mixed meal and a continuing morning fast. The study of these possible changes in leptin is important in view of the complex as well as circadian variations of plasma leptin [3]. Classically, leptin is not known to change in the postprandial period in response to a mixed meal and its circadian variations have not been related to any particular feeding patterns [3-5]. Moreover, little attention has been paid to the precise timing of fasting blood sampling in clinical studies done on leptin. We have examined these phenomena in three groups of volunteers. In all these experiments, immunoreactive leptin concentrations were assayed in duplicate using a commercially available radioimmunoassay (Linco Research, St. Louis, Mo., USA). All data are given as means \pm SEM.

In the first protocol, 18 subjects $(10 \mathrm{M} / 8 \mathrm{~W}$; mean age $55 \pm 2$; BMI $27.6 \pm 0.5 \mathrm{~kg} / \mathrm{m}^{2}$ ) with a normal glucose tolerance were studied with a $75-\mathrm{g}$ oral glucose tolerance test at 0800 hours in the fasting state. Leptin concentrations dropped by $-8.8 \pm 4.4 \%(p<0.001$; Wilcoxon signed rank test $) 2 \mathrm{~h}$ after the $75-\mathrm{g}$ oral glucose load. In a similar manner, the changes in leptin were also verified in 17 volunteers with newly diagnosed diabetes $\left(9 \mathrm{M} / 8 \mathrm{~F}\right.$; mean age $57 \pm 2$; BMI $\left.28.2 \pm 0.6 \mathrm{~kg} / \mathrm{m}^{2}\right)$ and 13 volunteers with impaired glucose tolerance according to World Health Organisation criteria $(8 \mathrm{M} / 5 \mathrm{~F}$; mean age $56 \pm$ 2 ; BMI $27.7 \pm 0.8 \mathrm{~kg} / \mathrm{m}^{2}$ ). The relative reduction of leptin was also statistically significant in both groups (diabetic $-10.6 \pm 1.9 \%$; IGT $-10.4 \pm 4.8 \%$ ) although there was no difference in magnitude between the three groups $(p>0.05)$. In the entire group $(n=48)$, women had much higher fasting leptin concentrations and a greater absolute reduction but the relative reduction of leptin was still $-11.7 \%$, a value no different from the one observed in men. Using an analysis of covariance that included sex and BMI (which can be important confounding variables in such a study), the reduction in plasma leptin concentration was no different between the three glucose tolerance groups $(p>0.05)$.

In a second protocol, a different group of 17 subjects $(10 \mathrm{M} /$ $7 \mathrm{~W}$; mean age $53 \pm 2$; BMI $28.9 \pm 0.7 \mathrm{~kg} / \mathrm{m}^{2}$ ), also with normal glucose tolerance, were studied similarly before and after the ingestion of $8.25 \mathrm{kcal} / \mathrm{kg}$ of a mixed breakfast meal composed of $28 \%$ fat, $12 \%$ protein and $60 \%$ carbohydrates. Here again, plasma leptin concentrations were measured before (0800 hours) and $2 \mathrm{~h}$ after the ingestion of this mixed meal. The relative reduction in leptin was of $-12.6 \pm 3.3 \%(p<0.02$; Wilcoxon signed rank test).

Finally, we studied a last group of 18 normal research volunteers $\left(10 \mathrm{M} / 8 \mathrm{~W}\right.$; mean age $48 \pm 2$; BMI $\left.27.3 \pm 1.0 \mathrm{~kg} / \mathrm{m}^{2}\right)$ who had agreed to continue their fast in the morning for 2 additional hours. Leptin concentrations were measured around 0800 hours and $2 \mathrm{~h}$ later. Under these conditions, plasma leptin concentrations dropped by $-9.4 \pm 3.9 \%(p<0.03$; Wilcoxon signed rank

Corresponding author: P. Maheux, M.D., Division of Endocrinology and Metabolism, Université de Sherbrooke, 3001 12th Avenue North, Sherbrooke, Quebec, Canada J1H 5N4 test). This relative reduction of plasma leptin was not statistically different from that observed in the other study groups.

In conclusion, there is a statistically significant reduction of plasma leptin concentrations when they are measured in the same individual $2 \mathrm{~h}$ apart in the morning, regardless of the underlying degree of glucose tolerance, fasting, or diet (mixed meal or drinking a $75-\mathrm{g}$ oral glucose tolerance test). This strongly suggests that important and significant circadian fluctuations of plasma leptin occur in fasting and non-fasting subjects in the morning. In clinical studies, these results should be very strong arguments for trying, when possible, to measure leptin concentrations at the same time in the morning. A statistically significant fluctuation of baseline plasma leptin of $10-12 \%$ over the course of $2 \mathrm{~h}$ in the morning can certainly obscure some small but significant changes in plasma leptin in short-term as well as in more long-term clinical studies. In addition, if it is also taken into account that plasma leptin has a 90min half life, it can be hypothesised that real changes in the release of leptin by adipose tissue are far more important over such a short period of time. Albeit the well established fact that leptin concentrations are higher in women and well correlated with BMI, our observations should draw attention to the existence of many variations among studies on changes in plasma leptin and on its correlation with various metabolic variables. As an example, the correlation between plasma insulin and leptin in the many papers that have been published on this matter is often inconsistent [6,7]. Although these differences are generally explained by the varying sizes of study groups and lack of statistical power, we would like to suggest that short-term and statistically significant circadian fluctuations of plasma leptin can occur if one does not take great care in attempting to measure plasma leptin at the same time in the morning. Finally, despite the known marked heterogeneity in leptin concentrations among people with similar body weight, the observed reduction of plasma leptin concentrations was, in our study, independent of sex or degree of glucose tolerance.

Yours sincerely,

D. Panarotto, P. Maheux

\section{References}

1. Kolaczynski JW, Nyce MR, Considine RV et al. (1996) Acute and chronic effect of insulin on leptin production in humans: studies in vivo and in vitro. Diabetes 45: 699-701

2. Malmström R, Taskinen M-R, Karonen SL, Yki-Jarvinen $H$ (1996) Insulin increases plasma leptin concentrations in normal subjects and patients with NIDDM. Diabetologia 39: 993-996

3. Sinha MK, Ohannesian JP, Heiman ML et al. (1996) Nocturnal rise of leptin in lean, obese and non-insulin dependent diabetes mellitus subjects. J Clin Invest 97: 1344-1347

4. Korbonits M, Trainer PJ, Little JA et al. (1997) Leptin levels do not change acutely with food administration in normal or obese subjects but are negatively correlated with pituitary-adrenal activity. Clin Endocrinol 46: 751-757

5. Karhunen L, Haffner S, Lappalainen R, Turpeinen A, Miettinen H, Uusitupa M (1997) Serum leptin and shortterm regulation of eating in obese women. Clin Science 92: $573-578$

6. Considine RV, Sinha MK, Heiman ML et al. (1996) Serum immunoreactive-leptin concentrations in normal weight and obese humans. N Engl J Med 334: 292-295

7. Zimmet P, Hodge A, Nicolson M et al. (1996) Serum leptin concentration, obesity, and insulin resistance in Western Samoans: cross sectional study. BMJ 313: 965-969 